\title{
GA BASED DIMENSIONALITY REDUCTION IN HYPERSPECTRAL IMAGE SEGMENTATION FRAMEWORK
}

\author{
B. Srinivas* \\ Research Scholar, Department of CS, Ralayaseema University, Kurnool, India \\ Dr. J. Rajendra Prasad \\ Professor, Department of IT, PVP Siddhartha Institute of Technology, Vijayawada, India \\ *Corresponding Author: B. Srinivas
}

\begin{abstract}
Hyperspectral imaging system contains stack of images collected from the sensor with different wavelengths representing the same scene on the earth. This paper presents GA based dimensionality reduction method in framework for hyperspectral image segmentation. The framework consists of four stages in segmenting a hyperspectral data set. In the first stage, filtering is done to remove noise in image bands. Second stage consists of dimensionality reduction algorithms, in which the bands that convey less information or redundant data will be removed. This deletion will decrease the storage requirement, computational load etc in processing the hyperspectral data. In the third stage, the informative bands which are selected in the second stage are merged into a single image using averaging method of fusion technique. The main goal of image fusion is to merge all the features from the selected image bands to form a single image. This single image is segmented using Fuzzy cmeans clustering algorithm. The experimental results show that this framework will segment the data set more accurately by combining all the features in the image bands after dimensionality reduction using proposed technique.
\end{abstract}

Key words: Image Processing, Hyperspectral Images, FCM, Remote Sensing.

Cite this Article: B. Srinivas and Dr. J. Rajendra Prasad, GA based Dimensionality Reduction in Hyperspectral Image Segmentation Framework, International Journal of Computer Engineering and Technology 10(4), 2019, pp. 1-9.

http://iaeme.com/Home/issue/IJCET?Volume $=10 \&$ Issue $=4$

\section{INTRODUCTION}

The process of information extraction about an object on the earth using satellites is called remote sensing [1]. With the increase of spatial and spectral resolution of recently launched satellites, new methods have to be developed in analyzing the remote sensing data. In remote sensing, sensors are available that can generate hyperspectral data, involving many narrow bands in which each pixel has a continuous reflectance spectrum [2]. Unsupervised image

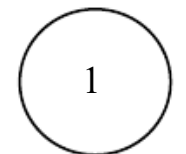


segmentation is an important research topic in hyperspectral imaging, with the aim to develop efficient algorithms that provide high segmentation accuracy. Existing methods for hyperspectral data sets is done by selecting limited number of bands normally less than seven. The accuracy of any segmentation algorithm decreases if the number of spectral bands increases. This paper presents a new methodology for dimensionality reduction in hyperspectral data set using GA. The framework for hyperspectral image segmentation consists of four stages noise removal, dimensionality reduction, image fusion and segmentation. The framework presented in this paper provides a methodology for segmenting the hyperspectral data set by incorporating all the information existing in the original bands rather than selecting some spectral bands. The frame work for hyperspectral image segmentation is shown in figure 1.

In the hyperspectral data set, some of the image bands contain noise which is caused by the sensor problems or disturbance of transmission medium in the atmosphere which affects result of image segmentation. To remove noise, many linear and non-linear filters are developed. In this paper, a new filter is designed based on Bi-dimensional Empirical Mode Decomposition [BEMD] and Mean filter. The BEMD method [3] decomposes the image band into several Intrinsic Mode Functions [IMF], in which the first function is the high frequency component, second function next high frequency component and so on, the last function denotes the low frequency component [5]. The mean filter is applied only to the few first high frequency components leaving the low frequency components, as the high frequency components contain noise. The image band is reconstructed by combining the filtered high frequency components and low frequency components. The same procedure is used for filtering the image bands.

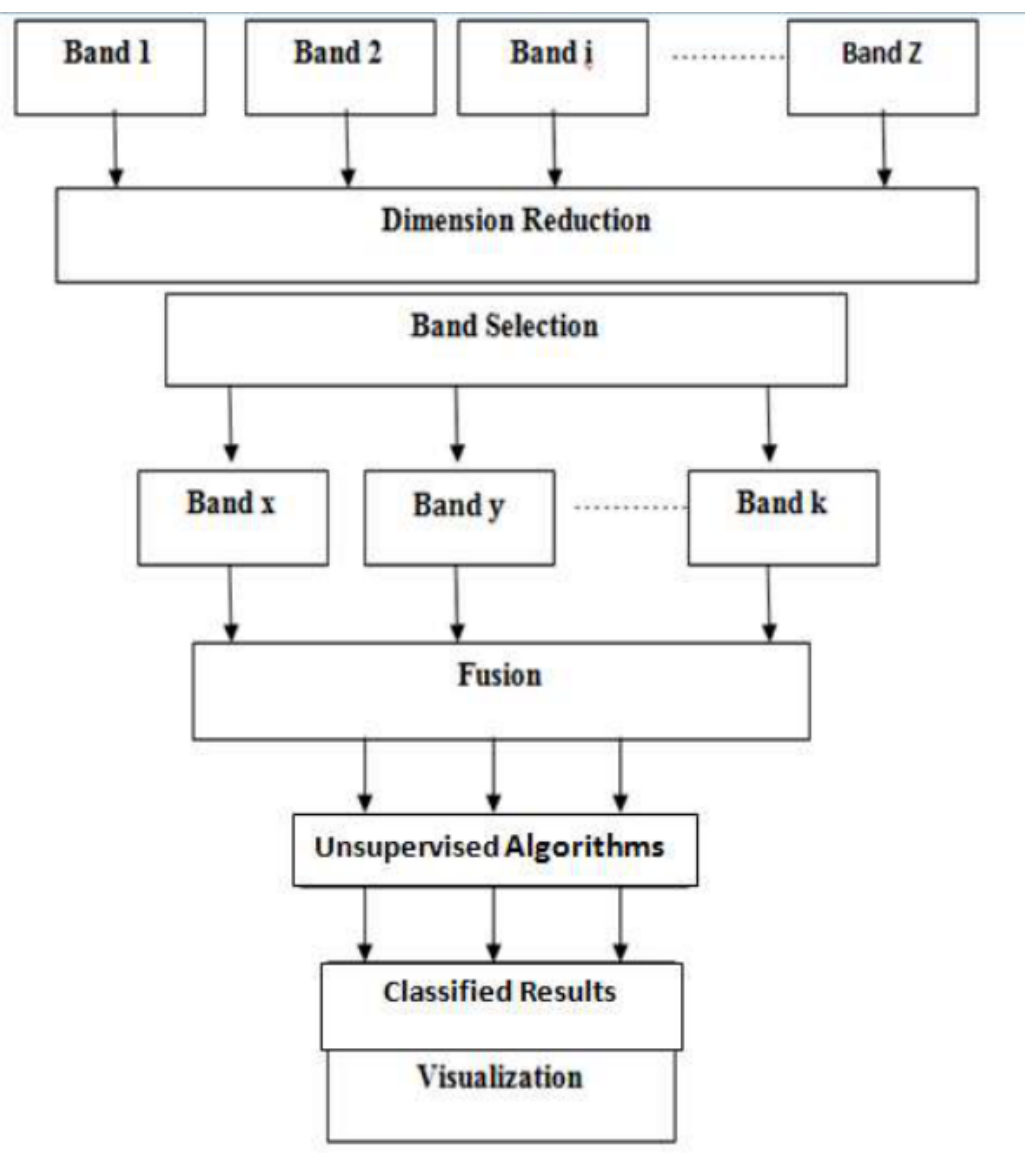

Figure 1 Hyperspectral Image Segmentation Framework 
This paper is structured as follows: section 2 presents GA based dimensionality reduction method, stage 3 presents image fusion technique using Averaging Method, section 4 presents FCM algorithm for image segmentation, section 5 shows experimental results and section 7 report conclusions.

\section{DIMENSIONALITY REDUCTION USING GA}

The filtering of bands is done using BEMD + Mean filtering algorithm presented in [7]. The dimensionality reduction can be done in two steps, feature extraction and band selection. The feature extraction methods retrieve the features in the original image bands to create a low dimension feature space. This feature extraction methods change the physical characteristics of the hyperspectral data set. On the other hand, the band selection methods select the best combination of image bands based on the information in the data set. The band selection methods are more suitable for dimensionality reduction of hyperspectral data sets than feature extraction methods. The dimensionality reduction step decreases many requirements for processing the hyperspectral data set such as storage space, computational load, communication bandwidth etc, thus increasing the efficiency of segmentation algorithm. This paper presents a new dimensionality reduction method using GA with the fitness function developed by combining the four band selection metrics such as Euclidean Distance [9], Spectral Angle Mapper [10], Spectral Correlation Mapper [11] and Band Correlation [11]. This four band selection metrics are defined as follows:

$$
\begin{gathered}
E D(X, Y)=\sqrt{\sum_{k=1}^{N}\left(X_{k}-Y_{k}\right)^{2}} \\
\operatorname{SAM}(X, Y)=\arccos \left(\frac{X^{T} . Y}{\|X\| \cdot\|Y\|}\right) \\
\operatorname{SCM}(X, Y)=\frac{\sum_{k=1}^{N_{b}}\left(X_{k}-\mu_{X}\right) \cdot\left(Y_{k}-\mu_{Y}\right)}{\left(N_{b}-1\right) \cdot \sigma_{X} \cdot \sigma_{Y}} \\
\operatorname{BC}(i, j)=\frac{\sum_{p=1}^{N_{b}}\left(x_{i p}-\mu_{i}\right) \cdot\left(x_{j p}-\mu_{j}\right)}{\sqrt{\sum_{p=1}^{N_{b}}\left(x_{i p}-\mu_{i}\right)^{2}} \cdot \sqrt{\sum_{p=1}^{N_{b}}\left(x_{j p}-\mu_{j}\right)^{2}}}
\end{gathered}
$$

Where $\mathrm{X}$ and $\mathrm{Y}$ are two vectors, $\mathrm{N}_{\mathrm{b}}$ denotes the number of bands, $\mu$ denotes the mean, $\sigma$ denotes the standard deviation of corresponding vector, $\mathrm{x}_{\mathrm{ip}}$ and $\mathrm{x}_{\mathrm{jp}}$ are the pixel value of bands $\mathrm{i}$ and $\mathrm{j}$, respectively. For ED and SAM bands with larger values are selected and for SCM and BC bands with smaller values are selected.

The genetic algorithm for band selection is described as follows:

a) Assume $\mathrm{P}$ chromosomes in the population where $\mathrm{P}$ is the size of the population. Each chromosome is a string of zeros and ones; the string length is equal to the number of bands in the data set. Zero bits in the string denote the corresponding band is not selected and one denotes the selection of specific band. In this method, we assume the total number of selected bands is fixed i.e., the total number of ones in each chromosome is fixed. 
b) Using an objective function, the fitness value of each chromosome is evaluated. The fitness function is defined as

$$
\text { fitnessfunction }=\frac{S C M * B C}{E D * S A M}
$$

c) The selection of chromosomes is done based on the fitness value using roulette wheel technique.

d) By applying single point crossover and mutation operators, a new population is produced from the parents. This new population replaces the old population. At any iteration the number of selected bands in each chromosome is fixed.

e) Maximum number of iterations is used as stopping criteria.

After the execution stops, the highest fitness value chromosome is selected and the values in this chromosome represent the solution to the band selection.

\section{IMAGE FUSION TECHNIQUE}

The hyperspectral data present abundant multidimensional information that contains far more image bands than those that can be displayed on the standard tristimulus display. Therefore, an efficient and appropriate means of visualization of the hyperspectral data is needed [12].

Let us consider $\mathrm{I}_{1}, \mathrm{I}_{2}, \ldots . \mathrm{I}_{\mathrm{k}}$ be a set of hyperspectral bands, containing $\mathrm{K}$ selected bands. We want to fuse these bands to generate a high contrast resultant image for visualization [4]. The primary aim of image fusion is to selectively merge the maximum possible features from the selected images to form a single image. Therefore, for an efficient fusion, we should be able to extract the specific information contained in a particular band [13].

The fused image $F$ at each stage can be represented as a linear combination of input images $\mathrm{I}_{\mathrm{k}}, \mathrm{k}=1$ to $\mathrm{M}$ as shown below:

$$
\begin{aligned}
& F(x, y)=\sum_{k=1}^{M} w_{k}(x, y) I_{k}(x, y) \\
& \text { and } \\
& \sum_{k=1}^{M} w_{i}(x, y)=1, \forall(x, y)
\end{aligned}
$$

where $\mathrm{w}_{\mathrm{k}}(\mathrm{x}, \mathrm{y})$ is the weight for the pixel at location $(\mathrm{x}, \mathrm{y})$ in the $\mathrm{k}$-th observation and $\mathrm{F}(\mathrm{x}, \mathrm{y})$ is the fused image. The weights are directly proportional to the finer details in the hyperspectral band

\section{FUZZY C-MEANS (UNSUPERVISED) ALGORITHM}

The FCM algorithm for segmentation of hyperspectral image is described below [14]:

- Take randomly $\mathrm{K}$ initial clusters from the $\mathrm{m}^{*} \mathrm{n}$ image pixels .

- Initialize membership matrix $u_{i j}$ with value in range 0 to 1 and value of $m=2$.

Assign each pixel to the cluster $\mathrm{Cj}\{\mathrm{j}=1,2, \ldots \ldots \mathrm{K}\}$ if it satisfies the following condition $[\mathrm{D}(.,)$. is the Euclidean distance measure between two values [6]].

$$
\begin{aligned}
& u_{i j}^{m} D\left(I_{i}, C_{j}\right)<u_{i q}^{m} D\left(I_{i}, C_{q}\right), q=1,2, \ldots, K \\
& j \neq q
\end{aligned}
$$

The new membership and cluster centroid values as calculated as 


$$
u_{i k}=\frac{1}{\sum_{j=1}^{K}\left(\frac{D\left(C_{i}, I_{k}\right)}{D\left(C_{j}, I_{k}\right)}\right)^{\frac{1}{m-1}}}, \text { for } 1 \leq i \leq K \quad C_{j}^{\wedge}=\frac{\sum_{j=1}^{n} u_{i j}^{m} I_{j}}{\sum_{j=1}^{n} u_{i j}^{m}}
$$

Continue 2-3 until each pixel is assigned to the maximum membership cluster [15].

\section{EXPERIMENTAL RESULTS}

The proposed methodology is tested on Pavia University and Indian Pines hyperspectral image data set collected from [16] containing 103 and 220 spectral bands. The dimensionality reduction is done by using four existing dimensionality metrics ED, SCM, SAM, BC and proposed method. After de-noising, from the Pavia university data set 60 best bands and from the Indian Pines data set 80 best bands were selected using proposed method. To compare the quantitative analysis of different dimensionality reduction methods, the entropy value is calculated after fusing the resultant bands obtained from each dimensionality reduction method using the average method. Table 1 shows the quantitative values of different dimensionality reduction methods. Table 1 shows that the proposed method of dimensionality reduction removes redundant bands efficiently. The fused image is segmented using FCM method in such a way that the grayscale intensity value of all the pixels in the image are grouped into nine clusters for Pavia University data set and sixteen clusters for Indian Pines data set. The qualitative analysis of the proposed method on Pavia University and Indian Pines hyperspectral data set is shown in figure 3.

Table 1 Quality of fused image after band selection

\begin{tabular}{|c|c|c|c|}
\hline \multicolumn{4}{|c|}{ Band Selection [After fusion using Average method] } \\
\hline $\begin{array}{c}\text { Method/India Pines } \\
\text { Data Set }\end{array}$ & Entropy & $\begin{array}{c}\text { Method/Pavia } \\
\text { University Data Set }\end{array}$ & Entropy \\
\hline ED & 4.91 & ED & 4.61 \\
\hline SAM & 4.96 & SAM & 4.78 \\
\hline SCM & 4.94 & SCM & 4.76 \\
\hline BC & 4.98 & BC & 4.81 \\
\hline PROPOSED & $\mathbf{5 . 0 9}$ & PROPOSED & $\mathbf{4 . 8 9}$ \\
\hline
\end{tabular}

\begin{tabular}{|c|c|c|}
\hline $\begin{array}{c}\text { Indian pines data set- image } \\
\text { band } 100\end{array}$ & IMF1 & IMF2 \\
\hline & & \\
\hline IMF3 & & $\begin{array}{c}\text { Image Band after de-noising } \\
\text { using IMF and Mean filter }\end{array}$ \\
\hline
\end{tabular}


B. Srinivas and Dr. J. Rajendra Prasad

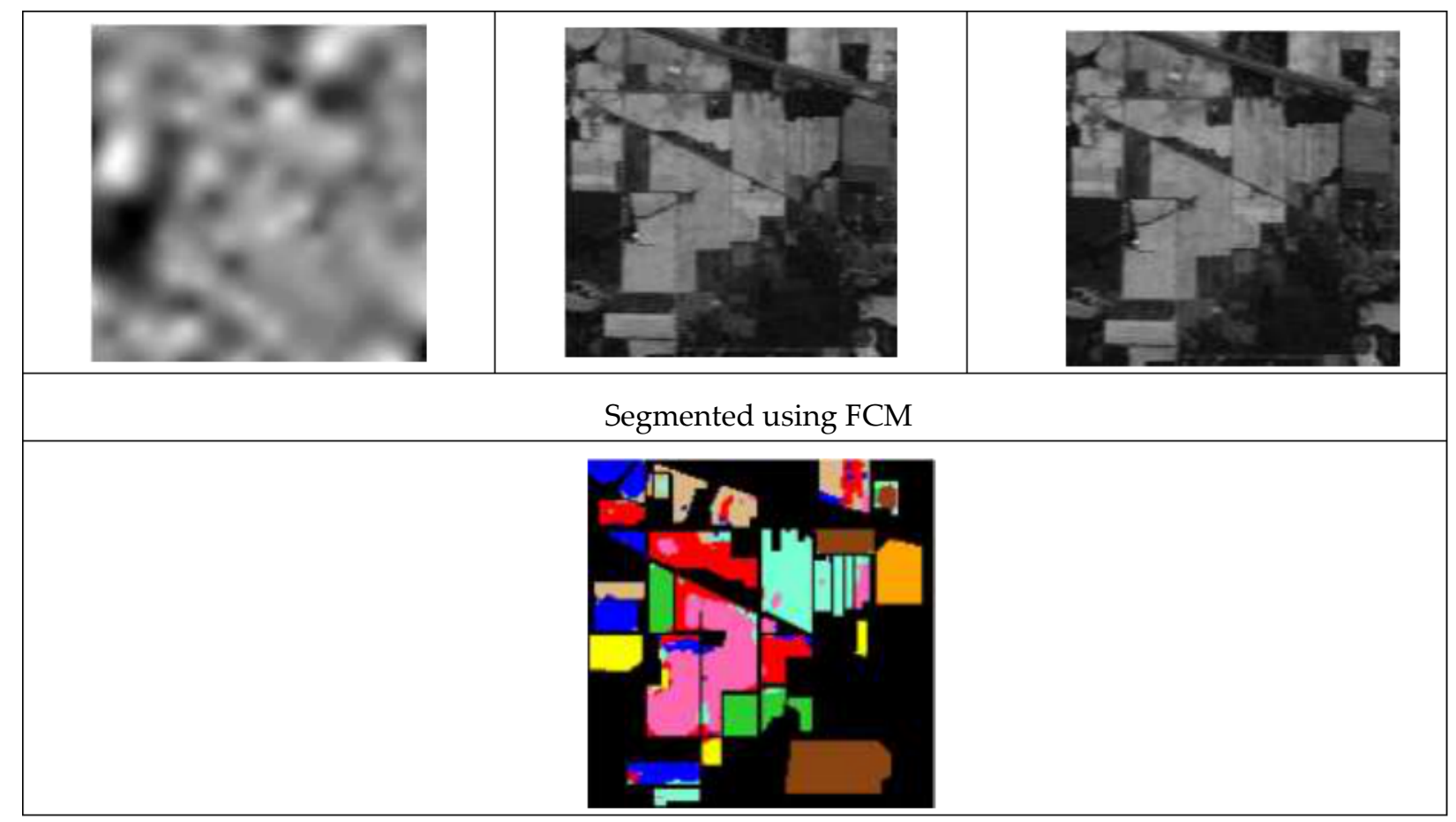

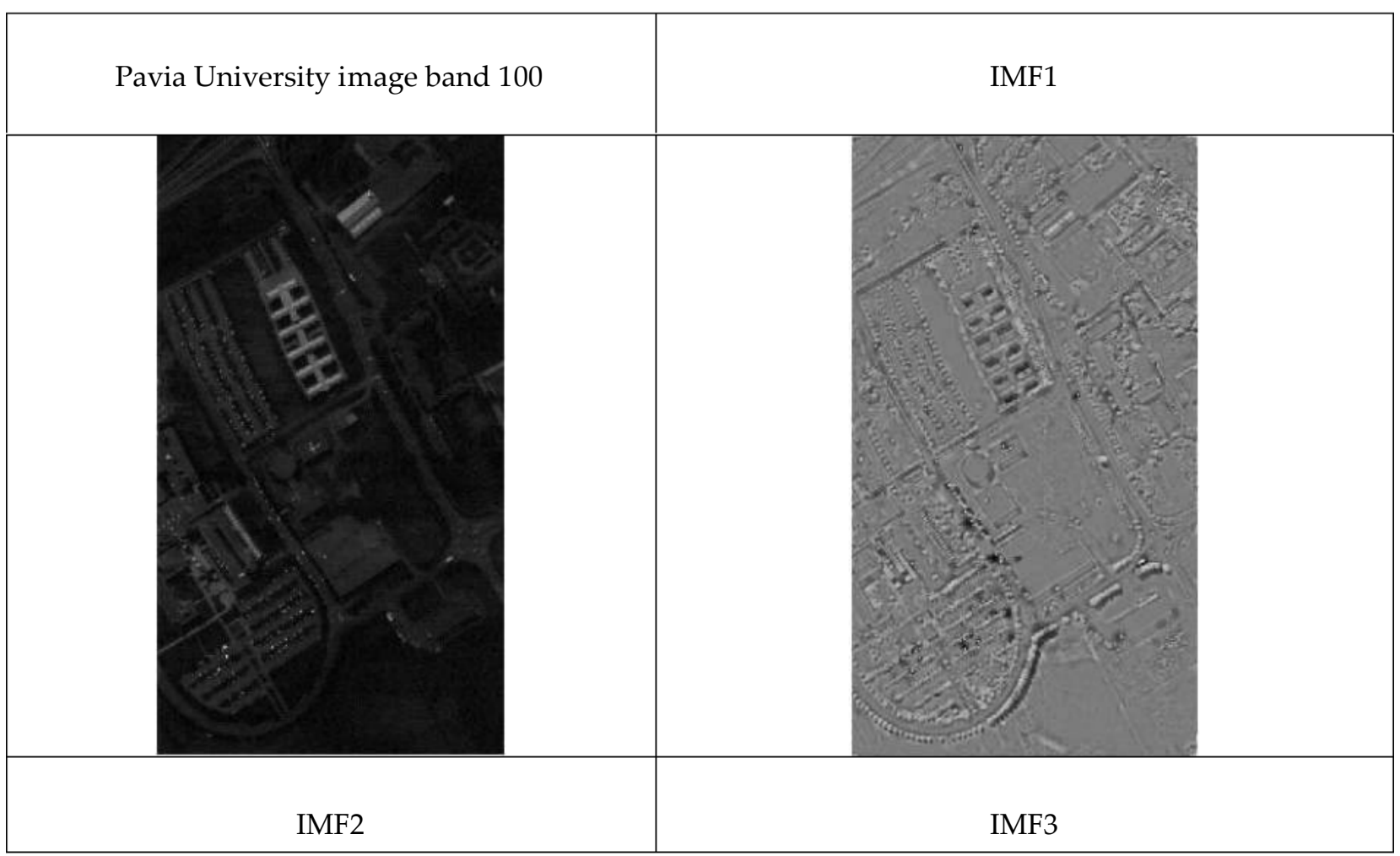




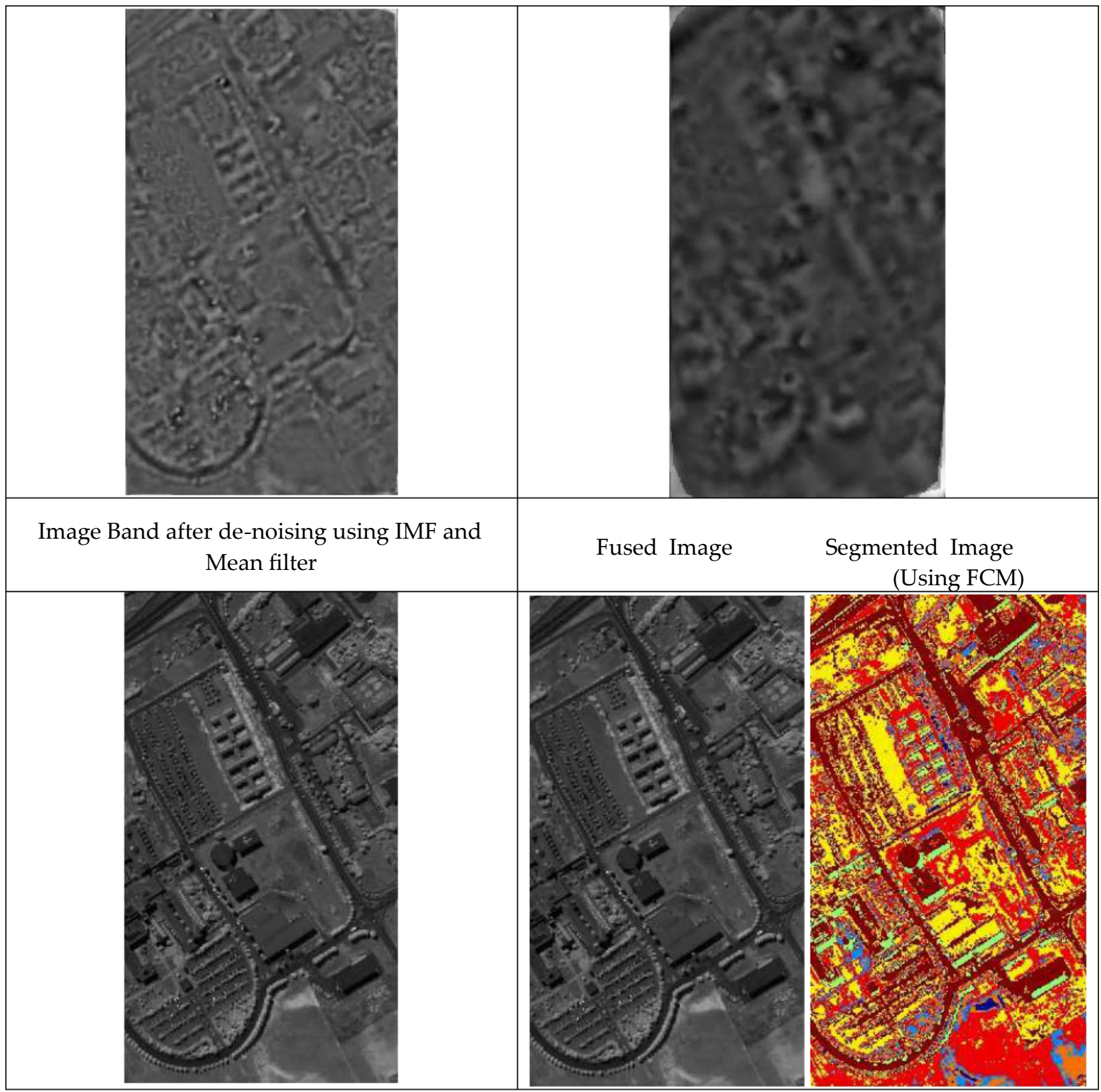

Figure 3 Hyperspectral Image segmentation

\section{CONCLUSIONS}

In this paper a framework for hyperspectral image segmentation is presented. The framework is carried out in four stages. First stage contains noise reduction algorithm in each image band, second stage contains dimensionality reduction using band selection methods to select informative bands leaving the bands that convey less descriptive information, third stage contains hierarchical image fusion to generate a single informative band and in the fourth stage, segmentation using FCM algorithm. Existing methods for hyperspectral data sets is done by selecting limited number of bands normally less than seven. The accuracy of any segmentation algorithm decreases if the number of spectral bands increases. In this paper, a new methodology of dimensionality reduction is presented using GA. The proposed methodology removes the redundant bands more efficiently than other dimensionality reduction bands. After dimensionality reduction, the selected bands are fused using averaging 
method and segmented using FCM. The framework presented in this paper provides a methodology for segmenting the hyperspectral data set by incorporating all the information existing in the original bands rather than selecting some spectral bands.

\section{REFERENCES}

[1] Yi Ma, Jie Zhang and Yufeng Gao (2008)," High Resolution Remote Sensing Image Classification of Coastal Zone and its Automatic Realization", Proceedings of IEEE International Conference on Computer Science and Software Engineering, pp. 827-829.

[2] B.Saichandana et.al. "Hyperspectral Image Enhancement Using Evolutionary Algorithm" International Journal of Advanced Research", Vol 3 Issue 4, March 2016 pp. no.934-938, 2016.

[3] J.Harikiran, et.al., "Spot Edge detection in Microarray Images using Bi-dimensional Empirical Mode Decomposition", C3IT-2012, AOT-WestBengal University, Proceedings in Elsevier Procedia Technology, (Science Direct) Volume 4, pp 19-25, 2012.

[4] Ketan Kotwal, et.al., "Visualization of Hyperspectral Images Using Bilateral Filtering",IEEE transactions of Geoscience and remote Sensing, Vol 48, No 5, May 2010, pp 2308-2319.

[5] J.Harikiran, et.al., "Fast Clustering Algorithms for Segmentation of Microarray Images", International Journal of Scientific \& Engineering Research, Volume 5, Issue 10, pp 569$574,2014$.

[6] Nor Ashidi Mat Isa, Samy A.Salamah, Umi Kalthum Ngah (2012)," Adaptive Fuzzy Moving K-means Clustering Algorithm for Image Segmentation", IEEE Transactions on Consumer Electronics, Vol. 55, Issue 4, pp 2145-2153.

[7] B.Saichandana et.al., "Clustering Algorithm combined with Empirical Mode decomposition for Classification of Remote sensing Image, "International Journal of Scientific and Engineering Research”, volume 5 Issue 9, September 2014, pp.686-695.

[8] B.Saichandana et.al., "Image Fusion in Hyperspectral Image Classification using Genetic Algorithm", Indonesian Journal of Electrical of Computer Engineering, volume 2 , No. 3, June 2016, pp.703-711.

[9] J. N. Sweet, "The spectral similarity scale and its application to the classification of hyperspectral remote sensing data," in Proc.Workshop Adv. Techn. Anal. Remotely Sensed Data,Washington, DC, Oct. 2003, pp. 92-99.

[10] B. Park, W. R. Windham, K. C. Lawrence, and D. P. Smith, "Contaminant classification of poultry hyperspectral imagery using a spectral angle mapper algorithm," Biosyst. Eng., vol. 96, no. 3, pp. 323-333, Mar. 2007.

[11] O. A. De Carvalho and P. R. Meneses, "Spectral correlation mapper (SCM): An improvement on the spectral angle mapper (SAM)," in Proc. Workshop Airborne Geoscience, Pasadena, CA, 2000.

[12] B.Saichandana, J.Harikiran, Dr.K.Srinivas, Dr.R.Kiran Kumar, "Application of BEMD and Hierarchical Image Fusion in Hyperspectral Image Classification", International Journal of Computer Science and Information Security, volume 14, number 5, May 2016, pp.437-445. 
GA based Dimensionality Reduction in Hyperspectral Image Segmentation Framework

[13] B.Guo, S.Gunn, B.Damper and J.Nelson,"Hyperspectral image fusion using spectrally weighted kernals", Proceedings of 8th International conference on Information Fusion, pp.402-408, 2005.

[14] J.Harikiran, et.al. "Multiple Feature Fuzzy C-means Clustering Algorithm for Segmentation of Microarray image", IAES International Journal of Electrical and Computer Engineering", Vol. 5, No. 5, pp. 1045-1053, 2015.

[15] J.Harikiran, et.al., "Fuzzy C-means with Bi-dimensional empirical Mode decomposition for segmentation of Microarray Image", International Journal of Computer Science issues, volume 9, Issue 5, Number 3, pp.273-279, 2012.

[16] http://www.ehu.eus/ccwintco/index.php?title=Hyperspectral_Remote_Sensing_Scenes.

[17] B.Saichandana, et.al, "Clustering Algorithm combined with Hill climbing for Classification of Remote Sensing Image", IAES International Journal of Electrical and Computer Engineering, volume 4, No 6, December 2014, pp.923-930. 